\title{
ON A VARIANT OF KORN'S INEQUALITY ARISING IN STATISTICAL MECHANICS
}

\author{
L. Desvillettes ${ }^{1}$ And CÉdRic Villani ${ }^{2}$
}

\begin{abstract}
We state and prove a Korn-like inequality for a vector field in a bounded open set of $\mathbb{R}^{N}$, satisfying a tangency boundary condition. This inequality, which is crucial in our study of the trend towards equilibrium for dilute gases, holds true if and only if the domain is not axisymmetric. We give quantitative, explicit estimates on how the departure from axisymmetry affects the constants; a MongeKantorovich minimization problem naturally arises in this process. Variants in the axisymmetric case are briefly discussed.
\end{abstract}

Mathematics Subject Classification. 49J40, 82C40, 76P05.

Received February 4, 2002.

\section{INTRODUCTION}

Korn's inequality asserts the control of the $L^{2}$ norm of the gradient of a vector field by the $L^{2}$ norm of just the symmetric part of this gradient, under certain conditions. Here is a rather general version: let $\Omega$ be a smooth bounded open set in $\mathbb{R}^{N}(N \geq 2$ to avoid trivial situations), then there exists a constant $\bar{K}(\Omega)>0$, such that for all vector fields $u: \Omega \rightarrow \mathbb{R}^{N}$,

$$
\left\|\nabla^{\mathrm{sym}} u\right\|_{L^{2}(\Omega)}^{2} \geq \bar{K}(\Omega) \inf _{R \in \mathcal{R}(\Omega)}\|\nabla(u-R)\|_{L^{2}(\Omega)}^{2}
$$

(see Friedrichs [6], Eq. (13), Second case, or Duvaut-Lions [5], Eq. (3.49)). Here $\nabla u$ and $\nabla^{\mathrm{sym}} u$ are matrixvalued applications defined by

$$
(\nabla u)_{i j}=\frac{\partial u_{i}}{\partial x_{j}}, \quad\left(\nabla^{\mathrm{sym}} u\right)_{i j}=\frac{1}{2}\left(\frac{\partial u_{i}}{\partial x_{j}}+\frac{\partial u_{j}}{\partial x_{i}}\right),
$$

and $\mathcal{R}(\Omega)$ stands for the finite-dimensional set of rigid motions on $\Omega$, i.e. affine maps $R: \Omega \rightarrow \mathbb{R}^{N}$ whose linear part is antisymmetric. Moreover, when $u=\left(u_{j}\right)$ and $M=\left(m_{i j}\right)$ are respectively a vector field and a matrix field on $\Omega$, we use the natural notations

$$
\|u\|_{L^{p}(\Omega)}=\left(\int_{\Omega}|u|^{p}\right)^{1 / p}, \quad\|M\|_{L^{p}(\Omega)}=\left(\int_{\Omega}|M|^{p}\right)^{1 / p},
$$

Keywords and phrases: Korn's inequality, Boltzmann equation, Monge-Kantorovich mass transportation problem.

1 Centre de Mathématiques et Leurs Applications, École Normale Supérieure de Cachan, 61 avenue du Président Wilson, 94235 Cachan, France; e-mail: desville@cmla.ens-cachan.fr

2 UMPA, École Normale Supérieure de Lyon, 69364 Lyon Cedex 07, France; e-mail: cvillani@umpa.ens-lyon.fr 
where

$$
|u|=\sqrt{\sum_{j=1}^{N} u_{j}^{2}}, \quad|M|=\sqrt{\sum_{i j} m_{i j}^{2}} .
$$

Note that $R$ is optimal in the right-hand side of (1) if and only if its linear part $\nabla R$ is just the average of the antisymmetric part of $\nabla u$ over $\Omega$.

Two commonly used variants of this inequality are the following:

$$
\|u\|_{L^{2}(\Omega)}^{2}+\left\|\nabla^{\mathrm{sym}} u\right\|_{L^{2}(\Omega)}^{2} \geq K^{\prime}(\Omega)\left(\|u\|_{L^{2}(\Omega)}^{2}+\|\nabla u\|_{L^{2}(\Omega)}^{2}\right),
$$

and

$$
\left.u\right|_{\Gamma}=0 \Longrightarrow\left\|\nabla^{\mathrm{sym}} u\right\|_{L^{2}(\Omega)}^{2} \geq K_{0}(\Omega)\|\nabla u\|_{L^{2}(\Omega)}^{2},
$$

where $\Gamma$ is a subset of $\partial \Omega$ with positive measure. Again, $K^{\prime}(\Omega)$ and $K_{0}(\Omega)$ are positive constants only depending on $\Omega$. When $\Gamma=\partial \Omega$, inequality (3) is very simple, as already noticed by Korn himself (see the remark in the Appendix). In all the other cases, inequalities (1-3) are much more delicate. We note that they still hold true if the $L^{2}$ norms are replaced by $L^{p}$ norms $(1<p<\infty)$. Also a more "global" variant of (1) was established in a famous study by Kohn [12]:

$$
\inf _{R \in \mathcal{R}(\Omega)}\|u-R\|_{L^{q}(\Omega)} \leq C_{p}(\Omega)\left\|\nabla^{\mathrm{sym}} u\right\|_{L^{p}(\Omega)},
$$

for any $p \in[1,+\infty), p \neq N$, with $q=N p /(N-p)(q=\infty$ if $p>N)$. Korn's inequality plays a fundamental role in elasticity theory (thinking of $u$ as a displacement vector field) and also in hydrodynamics (thinking of $u$ as a velocity vector field).

There is by now a huge literature on the subject: a research on the electronic database MathSciNet lists about 300 references directly concerned with Korn's inequality. Among the topics discussed there, let us only mention estimates of the best constants in certain situations (see for instance [2]), links with complex variable theory when $N=2$ (see for instance [11]), or generalizations to surfaces (see for instance [1], Vol. III). Ciarlet [1] (Vol. I, p. 291) enumerates about half a dozen proofs of Korn's inequality, one of which is detailed, and provides background on its applications. Horgan [10] summarizes the major known results for bounded domains in two and three dimensions, with emphasis on the estimates of the constants.

Korn's original proofs [13] were considered somewhat obscure, and many authors have endeavored to give simplified and improved arguments. Gobert [8] has proven (2) with the help of the theory of singular integral operators. The name of J.-L. Lions is attached to a particularly elegant and robust proof ([5], Sect. 3.3), which we will recall below. An elementary constructive proof of (2), based on extension operators, has been given by Nitsche [14]. We also mention Oleinik's beautiful argument [15] towards (2), based on a clever use of elementary estimates for harmonic functions and Hardy inequalities.

Let us here briefly recall Lions' argument [5] towards (1) (actually, a very slight variation of it). It is based on the following two lemmas. The first one has been known since immemorial times, while the second is part of the theory of distributions.

Lemma 1. Let $u \in H^{1}\left(\Omega ; \mathbb{R}^{N}\right)$. Then, for all $i, j, k \in\{1, \ldots, N\}$,

$$
\frac{\partial^{2} u_{k}}{\partial x_{i} \partial x_{j}}=\frac{\partial}{\partial x_{i}}\left(\nabla^{\mathrm{sym}} u\right)_{j k}+\frac{\partial}{\partial x_{j}}\left(\nabla^{\mathrm{sym}} u\right)_{i k}-\frac{\partial}{\partial x_{k}}\left(\nabla^{\mathrm{sym}} u\right)_{i j} .
$$


In this lemma, the notation $H^{1}(\Omega)$ stood for the usual Sobolev space defined by the norm $\|f\|_{H^{1}}^{2}=\|f\|_{L^{2}}^{2}$ $+\|\nabla f\|_{L^{2}}^{2}$, and derivatives were taken in distributional sense. From Lemma 1 we only retain the

Corollary 1. Each partial derivative of each component of $\nabla u$ can be written as a linear combination of partial derivatives of components of $\nabla^{\mathrm{sym}} u$.

In short, $\nabla \nabla u$ is a "matrix combination" of $\nabla \nabla^{\mathrm{sym}} u$. For the next lemma, we shall introduce the notation

$$
\langle f\rangle=\frac{1}{|\Omega|} \int_{\Omega} f
$$

where $|\Omega|$ stands for the $N$-dimensional volume of $\Omega$. Of course $\langle f\rangle$ is just the $L^{2}$ projection of $f$ onto the space of constant functions. We also define the $H^{-1}$ norm of a given function (or distribution) $f$ in $\Omega$ by

$$
\|f\|_{H^{-1}(\Omega)}=\sup \left\{\int_{\Omega} f \varphi ; \varphi \in \mathcal{D}(\Omega),\|\nabla \varphi\|_{L^{2}(\Omega)} \leq 1\right\},
$$

where $\mathcal{D}(\Omega)$ stands for the space of $C^{\infty}$ functions with compact support in $\Omega$. When $v$ is an $L^{2}$ vector field on $\Omega$, we naturally define

$$
\|v\|_{H^{-1}(\Omega)}^{2}=\sum_{i=1}^{N}\left\|v_{i}\right\|_{H^{-1}(\Omega)}^{2}
$$

Then one has the

Lemma 2. There exists a constant $C(\Omega)$, only depending on $\Omega$, such that for all $f \in L^{2}(\Omega)$,

$$
\|\nabla f\|_{H^{-1}(\Omega)}^{2} \leq N\|f-\langle f\rangle\|_{L^{2}(\Omega)}^{2} \leq C(\Omega)\|\nabla f\|_{H^{-1}(\Omega)}^{2} .
$$

Corollary 2. Let $f$ and $g_{i j}(1 \leq i \leq N, 1 \leq j \leq N)$ be $L^{2}$ real-valued functions on $\Omega$, such that for all $i$,

$$
\frac{\partial f}{\partial x_{i}}=\sum_{j} \alpha_{i j} \frac{\partial g_{i j}}{\partial x_{j}}
$$

Then

$$
\|f-\langle f\rangle\|_{L^{2}}^{2} \leq N^{2} C(\Omega)\left(\sup _{i j}\left|\alpha_{i j}\right|^{2}\right) \sum_{i j}\left\|g_{i j}\right\|_{L^{2}(\Omega)}^{2} .
$$

Note that the constant $C(\Omega)$ in the above formula is invariant by dilation of $\Omega$, but has to depend on the shape of the domain, as can be seen by looking at the case when $\Omega$ is very elongated in one direction. For instance, in dimension $N=2$, choose $\Omega=\left\{\left(x_{1}, x_{2}\right) \in \mathbb{R}^{2} ;\left(\varepsilon x_{1}\right)^{2}+\left(x_{2} / \varepsilon\right)^{2} \leq 1\right\}$. By considering $f(x)=x_{1}, g_{12}(x)=x_{2}$, $g_{i j}=0$ else, one immediately sees that $C(\Omega) \rightarrow+\infty$ as $\varepsilon \rightarrow 0$.

The first inequality in (5) is readily obtained by integration by parts, and only the second one is tricky. It can be shown by closed graph theorem, or by the construction of an appropriate extension operator. The variant which is explicitly proven in [5] is

$$
\|f\|_{L^{2}} \leq C\left(\|f\|_{H^{-1}}+\|\nabla f\|_{H^{-1}}\right)
$$

We also give the sketch of a simple, constructive proof communicated to us by Meyer. Denoting by $\Delta^{-1}$ the bijective operator from $H^{-1}(\Omega)$ to $H_{0}^{1}(\Omega)$ corresponding to the solution of the Laplace problem on $\Omega$ with 
Dirichlet boundary condition, one has

$$
\Delta f=\Delta\left[\sum_{j=1}^{N} \partial_{j} \Delta^{-1}\left(\partial_{j} f\right)\right] .
$$

Here we use the shorthand $\partial_{j}=\partial / \partial x_{j}$. In particular,

$$
\|f\|_{L^{2}(\Omega)} \leq\left\|\sum_{j=1}^{N} \partial_{j} \Delta^{-1} \partial_{j} f\right\|_{L^{2}(\Omega)}+\left\|w_{f}\right\|_{L^{2}(\Omega)},
$$

where $w_{f}$ is harmonic on $\Omega$. But there exists a constant $C$, only depending on $\Omega$, such that

$$
\left\|\sum_{j=1}^{N} \partial_{j} \Delta^{-1} \partial_{j} f\right\|_{L^{2}(\Omega)} \leq C \sum_{j=1}^{N}\left\|\partial_{j} f\right\|_{H^{-1}(\Omega)} ;
$$

so it is sufficient to prove Lemma 2 for harmonic functions on $\Omega$. Thus, let $f$ be a harmonic function on $\Omega$. Of course we have

$$
\|f-\langle f\rangle\|_{L^{2}(\Omega)} \leq\left\|f-\langle f\rangle_{\partial \Omega}\right\|_{L^{2}(\Omega)},
$$

where $\langle f\rangle_{\partial \Omega}$ stands for the average of the trace of $f$ on $\partial \Omega$. But, since $\Omega$ is smooth and connected, there exists an isomorphism between harmonic functions in $H^{s}(\Omega)$ and their traces in $H^{s-1 / 2}(\partial \Omega$ ), for all $s \in \mathbb{R}$ (here we only need $s=0$ and $s=-1$ ). So we just have to prove

$$
\left\|f-\langle f\rangle_{\partial \Omega}\right\|_{H^{-1 / 2}(\partial \Omega)} \leq C\|\nabla f\|_{H^{-3 / 2}(\partial \Omega)} .
$$

And since $\partial \Omega$ is a smooth set without boundary, the proof can be carried out by use of local charts, reduction to $\mathbb{R}^{N-1}$ and Fourier transform.

It remains to understand why Corollaries 1 and 2 together imply (1). For this, let $\nabla^{\mathrm{a}} u$ stand for the antisymmetric part of $\nabla u$,

$$
\left(\nabla^{\mathrm{a}} u\right)_{i j}=\frac{1}{2}\left(\frac{\partial u_{i}}{\partial x_{j}}-\frac{\partial u_{j}}{\partial x_{i}}\right)
$$

From Corollaries 1 and 2 it follows that $\left\|\nabla^{\mathrm{a}} u-\left\langle\nabla^{\mathrm{a}} u\right\rangle\right\|_{L^{2}(\Omega)}$ is bounded by a constant multiple of $\left\|\nabla^{\mathrm{sym}} u\right\|_{L^{2}(\Omega)}$. Then (1) is a consequence of

$$
\left\|\nabla u-\left\langle\nabla^{\mathrm{a}} u\right\rangle\right\|^{2}=\left\|\nabla^{\mathrm{sym}} u\right\|_{L^{2}(\Omega)}^{2}+\left\|\nabla^{\mathrm{a}} u-\left\langle\nabla^{\mathrm{a}} u\right\rangle\right\|_{L^{2}(\Omega)}^{2} .
$$

\section{Motivation AND MAIN RESUlt}

We shall now explain our interest in Korn's inequality. The present work was not motivated by elasticity or hydrodynamics, but by a different area of applications, namely statistical physics, and more precisely the kinetic theory of rarefied gases. Let us sketch the problem.

Since the works of Maxwell and Boltzmann more than a hundred years ago, it has been admitted by physicists that a gas enclosed in a bounded box, undergoing appropriate boundary interaction, should approach a certain 
steady state as time becomes large. Here the gas is modelled by the Boltzmann equation, which is supposed to accurately describe collisions inside a dilute gas. This steady state would achieve a maximum of the entropy under the constraints imposed by the physical conservation laws. And at least for generic shape of the box, it would be a rest state, in the sense that the density and temperature would be constant all over the box, and that there would be no macroscopic velocity field. Such a statement cannot be true for all boxes: in fact, when the box has cylindrical shape, and specular boundary condition is enforced (meaning that particles just bounce on the boundary of the box according to the Snell-Descartes laws), then there are steady states which are not at rest, and possess a "rotating" velocity field. This does not contradict the principle of maximum entropy, because the presence of an axis of symmetry induces an additional conservation law, namely the conservation of a coordinate of the angular momentum. In all other realistic situations (at least when the boundary conditions do not depend on time), it is expected that the distribution of particles does converge towards a rest state.

The mathematical justification of this guess is rather easy as soon as suitable a priori bounds on solutions of the Boltzmann equation have been obtained. Such bounds are not trivial at all, and at present seem to have been established only in a close-to-equilibrium setting for a convex box (this was achieved in the seventies, see for instance Shizuta and Asano [18]). But once they are settled, then the result of trend to equilibrium is an immediate consequence of the classification of steady states (see for instance Desvillettes [3]) and an elementary compactness argument.

Now, what turns out to be much more complicated is to get a quantitative result of convergence to equilibrium, with explicit rates of convergence. By this we mean the following: let be a solution of the Boltzmann equation, not necessarily close to equilibrium, satisfying "natural" a priori estimates, uniform in time, then can one find explicit estimates on how fast it converges towards equilibrium? Among the main causes for this tremendous increase of difficulty are the intricate nature of the Boltzmann collision operator, the fact that it admits three conservation laws (mass, momentum and energy) and the degenerate nature of the Boltzmann equation with respect to the position variable.

In a work in progress [4], we overcome these three difficulties, and obtain explicit rates of convergence to equilibrium for solutions of the Boltzmann equation satisfying certain strong a priori estimates (smoothness, decay at infinity, strict positivity). One of the many steps in that work consists in expressing how much the domain deviates from axisymmetry, in a way which can be used to estimate rates of convergence. By convention, we say that a domain in $\mathbb{R}^{2}$ is axisymmetric if it has a circular symmetry around some point; and that a domain in $\mathbb{R}^{3}$ is axisymmetric if it admits an axis of symmetry (which means that it is preserved by a rotation of arbitrary angle around this axis). By abuse of language, for any $N \geq 4$, we shall say that a domain is axisymmetric if it is left invariant by the action of a one-parameter group $\left(\mathrm{e}^{t R}\right)_{t \in \mathbb{R}}$, where $R$ is an affine map with antisymmetric linear part (see Definition-Lemma 1). It turned out, to our surprise, that the degree of non-axisymmetry of the domain $\Omega$ could be expressed by means of the following Korn-like inequality.

Theorem 3. Let $\Omega$ be a $C^{1}$ bounded, non-axisymmetric open subset of $\mathbb{R}^{N}(N \geq 2)$. Let $u$ be a vector field on $\Omega$ with $\nabla u \in L^{2}(\Omega)$. Assume that $u$ is tangent to $\partial \Omega$ :

$$
\forall x \in \partial \Omega, \quad u(x) \cdot n(x)=0,
$$

where $n(x)$ stands for the outer unit normal vector to $\Omega$ at point $x$. Then there exists a constant $K(\Omega)>0$, only depending on $\Omega$, such that

$$
\left\|\nabla^{\mathrm{sym}} u\right\|_{L^{2}(\Omega)}^{2} \geq K(\Omega)\|\nabla u\|_{L^{2}(\Omega)}^{2}
$$

There are two points to be made about Theorem 3. First, as we already mentioned, it is only via the boundary conditions that it differs from more standard versions of Korn's inequality, like (3) for instance. Indeed, usually one would impose that $u$ vanishes on $\partial \Omega$, or at least part of it. In the context of hydrodynamics, this corresponds to the well-known "no-slip" boundary condition; in elasticity, this reflects the usual assumption that part of the elastic body is attached to some region of the physical space. Apart from the present work, Ryzhak's paper $[16]$ is the only one known to us which has been interested in tangency boundary conditions. From the point of 
view of fluid dynamics, our context of application may seem rather strange, because $\left\|\nabla^{\mathrm{sym}} u\right\|_{L^{2}}^{2}$ looks like an energy dissipation term, of the kind encountered in the theory of the Navier-Stokes equations; but the tangency boundary condition on $u$ is typical of inviscid models, like the Euler equation. There is no contradiction at the level of the modelling, because in our method the term $\left\|\nabla^{\mathrm{sym}} u\right\|_{L^{2}}^{2}$ is not obtained as a dissipation term, but as the leading order, in some sense, of the second derivative of a certain functional.

The second point on which we attract the attention of the reader is the importance which we give to the value of the positive constant $K(\Omega)$ in (8). In our study of trend to equilibrium, the value of the constant $K(\Omega)$ is used to quantify the deviation of $\Omega$ from axisymmetry. It is therefore of great interest to have as much insight as possible in the explicit value of $K(\Omega)$, in terms of the geometry of $\Omega$. In fact, the main interest of the present work is to provide the following estimates on $K(\Omega)$.

Theorem 3 (continued). The largest admissible constant $K(\Omega)$ in (8) satisfies

$$
K(\Omega)^{-1} \leq 2 N\left(1+C_{H}(\Omega)\right)\left(1+\bar{K}(\Omega)^{-1}\right)\left(1+G(\Omega)^{-1}\right)
$$

where the various constants above are defined as follows:

- $C_{H}=C_{H}(\Omega)$ is a constant related to the homology of $\Omega$ and the Hodge decomposition, defined by the inequality

$$
\left\|\nabla^{\mathrm{sym}} v\right\|_{L^{2}(\Omega) / V_{0}(\Omega)}^{2} \leq C_{H}\left(\|\nabla \cdot v\|_{L^{2}(\Omega)}^{2}+\left\|\nabla^{\mathrm{a}} v\right\|_{L^{2}(\Omega)}^{2}\right),
$$

or (almost) equivalently by inequality (13) below. Here $\nabla \cdot v$ stands for the divergence of the vector field $v, \nabla \cdot v=\sum_{i} \partial v_{i} / \partial x_{i}$, and $V_{0}(\Omega)$ is the space of all vector fields $v_{0} \in H^{1}\left(\Omega ; \mathbb{R}^{N}\right)$ such that

$$
\nabla \cdot v_{0}=0, \quad \nabla^{\mathrm{a}} v_{0}=0 .
$$

We recall that $V_{0}$ is a finite-dimensional vector space whose dimension depends only on the topology of $\Omega$;

- $\bar{K}(\Omega)$ is the constant in $(1)$;

- and finally, $G=G(\Omega)$ is what we shall call Grad's number:

$$
G(\Omega)=\frac{1}{2|\Omega|} \inf _{\Sigma \in U A_{N}} \inf _{v \in V_{\Sigma}}\left\|\nabla^{\mathrm{sym}} v\right\|_{L^{2}(\Omega)}^{2} .
$$

Here $U A_{N}$ is the space of antisymmetric $N \times N$ real matrices with unit norm:

$$
\Sigma \in U A_{N} \Longleftrightarrow\left(\Sigma+{ }^{T} \Sigma=0 \quad \text { and } \quad|\Sigma|=1\right)
$$

and for any $N \times N$ matrix $\Sigma$, we define $V_{\Sigma}$ as the set of all vector fields in $H^{1}(\Omega)$ satisfying

$$
\begin{cases}\nabla \cdot v \equiv 0, & \nabla^{\mathrm{a}} v \equiv \Sigma \quad \text { in } \Omega \\ v \cdot n=0 & \text { on } \partial \Omega .\end{cases}
$$

Moreover, $G(\Omega)>0$ and, at least when $N=2$ or 3, an explicit lower bound on $G(\Omega)$ can be given in terms of "basic" geometrical information about how far $\Omega$ is from being axisymmetric. 


\section{Remarks.}

1. In dimension $N=3$, one can identify the space $A_{3}$ of $3 \times 3$ antisymmetric matrices to $\mathbb{R}^{3}$ in the usual way, via $\Sigma x=\sigma \wedge x$. Then, to any $\Sigma \in U A_{3}$ is associated $\sigma \in S^{2}$ such that

$$
\Sigma x=\frac{\sigma \wedge x}{\sqrt{2}} .
$$

One then recovers (up to a factor $|\Omega|$ ) the formula which appears in Grad ([9], p. 274):

$$
G(\Omega)=\frac{1}{|\Omega|} \inf _{\sigma \in S^{2}} \inf _{v \in V_{\sigma}}\left\|\nabla^{\mathrm{sym}} v\right\|_{L^{2}(\Omega)}^{2},
$$

where $V_{\sigma}$ is defined by the equations

$$
\nabla \cdot v=0, \quad \nabla \wedge v=\sigma, \quad v \cdot n=0 \quad \text { on } \partial \Omega .
$$

Of course $\nabla \wedge v$ is the curl of $v$. Also when $N=2$, one can identify $U A_{2}$ with $S^{0}=\{-1,+1\}$.

2. Grad may not have been the first one to consider the quantity $G(\Omega)$, but most probably he was the first one to understand that this number may be useful in the context of the Boltzmann equation. Even more, to our knowledge his paper is the only one to mention this fact. This justifies our terminology of "Grad's number". The present work drew a lot of inspiration from Grad's paper [9], which is at the same time quite obscure, definitely false and really illuminating in certain respects - as we will discuss in [4].

3. If $\Omega$ is simply connected, which is presumably the most natural case for applications, then $V_{0}=0$ and $V_{\Sigma}$ contains a unique element (we shall show in a moment that $V_{\Sigma}$ is never empty).

4. Our primary goal was to obtain fully explicit lower bounds for $K(\Omega)$ in terms of simple geometrical information about $\Omega$; to achieve this completely with our method, we would have to give quantitative estimates on $C_{H}$. Unfortunately, we have been unable to find explicit estimates about $C_{H}$ in the literature, although it seems unlikely that nobody has been interested in this problem. Of course, when $N=3$ and $\Omega$ is simply connected, estimate (10) is equivalent to

$$
\|\nabla u\|_{L^{2}(\Omega)}^{2} \leq C_{H}(\Omega)\left(\|\nabla \cdot u\|_{L^{2}(\Omega)}^{2}+\|\nabla \wedge u\|_{L^{2}(\Omega)}^{2}\right),
$$

up to possible replacement of $C_{H}$ by $C_{H}+1$. This is an estimate which is well-known to many people, but for which it seems very difficult to find an accurate reference. Inequality (10) can be seen as a consequence of the closed graph theorem; for instance, in the case of a simply connected domain, one just needs to note that (i) $\left\|\nabla^{\mathrm{a}} u\right\|_{L^{2}}^{2}+\|\nabla \cdot u\|_{L^{2}}^{2}$ is bounded by $\|\nabla u\|_{L^{2}}^{2}$, (ii) the identities $\nabla \cdot u=0, \nabla^{\mathrm{a}} u=0, u \cdot n=0$ (on the boundary), together imply $u=0$; so in fact the norms appearing on the left and on the right-hand side of (10) have to be equivalent. The proof of point (ii) is as follows: from Poincaré's lemma in a simply connected domain, there exists a real-valued function $\psi$ such that $\nabla \psi=u$; then $\psi$ is a harmonic function with homogeneous Neumann boundary condition, so it has to be a constant, and $u=0$.

Of course this argument gives no insight on how to estimate the constants. As pointed out to us independently by Druet and by Serre, one can choose $C_{H}(\Omega)=1$ if $\Omega$ is convex, but the general case seems to be much harder. Anyway this is a separate issue which has nothing to do with axisymmetry; all the relevant information about axisymmetry lies in our estimates on $G(\Omega)^{-1}$.

The organization of the paper is as follows: after a short proof of Theorem 3 in Section 3, we shall give some quantitative estimates on the positivity of $G(\Omega)$ in Section 4, and finally give a brief discussion of the axisymmetric case in Section 5. In an Appendix, we reproduce a proof of the abovementioned estimate of $C_{H}$ when $\Omega$ is convex, which was communicated to us by Druet. 


\section{Proof of Theorem 3}

To begin with, let us check that Definition 11 makes sense.

Lemma 4. For any $\Sigma \in U A_{N}$, the set $V_{\Sigma}$ is not empty.

Proof. Let $\Sigma \in U A_{N}$, and let $\varphi$ be a solution of the Laplace problem

$$
\left\{\begin{array}{l}
\Delta \varphi=0 \quad \text { in } \Omega \\
\nabla \varphi \cdot n=-\Sigma \cdot n \quad \text { on } \partial \Omega
\end{array}\right.
$$

The existence of $\varphi$ is ensured by the identity $\int_{\Gamma} n(x) \mathrm{d} \sigma(x)=0$ for each connected part $\Gamma$ of $\partial \Omega$. Then define $v(x)=\nabla \varphi(x)+\Sigma x$; one easily checks that $v \in V_{\Sigma}$.

Remark. When $\Omega$ is simply connected, this is the only solution.

We now proceed to prove Theorem 3 .

Proof of Theorem 3. Let us start from inequality (1), in the form

$$
\left\|\nabla^{\mathrm{a}} u-\left\langle\nabla^{\mathrm{a}} u\right\rangle\right\|_{L^{2}(\Omega)}^{2}+\left\|\nabla^{\mathrm{sym}} u\right\|_{L^{2}(\Omega)}^{2} \leq \bar{K}(\Omega)^{-1}\left\|\nabla^{\mathrm{sym}} u\right\|_{L^{2}(\Omega)}^{2} .
$$

If $\left\langle\nabla^{\mathrm{a}} u\right\rangle=0$, then we are done. If not, introduce

$$
\Sigma=\frac{\left\langle\nabla^{\mathrm{a}} u\right\rangle}{\left|\left\langle\nabla^{\mathrm{a}} u\right\rangle\right|} \in U A_{N}, \quad \lambda=\left|\left\langle\nabla^{\mathrm{a}} u\right\rangle\right|>0 .
$$

Let $v \in V_{\Sigma}$, then

$$
\left\{\begin{array}{l}
\nabla \cdot(\lambda v)=0, \quad \nabla^{\mathrm{a}}(\lambda v)=\left\langle\nabla^{\mathrm{a}} u\right\rangle \quad \text { in } \Omega \\
v \cdot n=0 \quad \text { on } \partial \Omega,
\end{array}\right.
$$

so that (15) implies

$$
\int_{\Omega}\left|\nabla^{\mathrm{a}}(u-\lambda v)\right|^{2} \leq \bar{K}(\Omega)^{-1}\left\|\nabla^{\mathrm{sym}} u\right\|_{L^{2}(\Omega)}^{2}
$$

and

$$
\int_{\Omega}\left|\nabla^{\mathrm{a}}(u-\lambda v)\right|^{2}+\int_{\Omega}|\nabla \cdot(u-\lambda v)|^{2} \leq N \bar{K}(\Omega)^{-1}\left\|\nabla^{\mathrm{sym}} u\right\|_{L^{2}(\Omega)}^{2} .
$$

In particular,

$$
\begin{aligned}
\int_{\Omega}\left|\nabla^{\mathrm{a}} u\right|^{2} & \leq 2 \int_{\Omega}\left|\nabla^{\mathrm{a}}(u-\lambda v)\right|^{2}+2 \lambda^{2} \int_{\Omega}\left|\nabla^{\mathrm{a}} v\right|^{2} \\
& \leq 2 \bar{K}(\Omega)^{-1}\left\|\nabla^{\mathrm{sym}} u\right\|_{L^{2}(\Omega)}^{2}+2 \lambda^{2}|\Omega|
\end{aligned}
$$

(recall that $\left|\nabla^{\mathrm{a}} v\right| \equiv 1$ ). 
To conclude the proof of Theorem 3, it only remains to bound $|\Omega| \lambda^{2}$ in terms of $\left\|\nabla^{\mathrm{sym}} u\right\|_{L^{2}}^{2}$. This is the point where Grad's number will show up! From (10) and (17) we know that there exists $w_{0} \in V_{0}$ such that

$$
\begin{aligned}
\int_{\Omega}\left|\nabla^{\mathrm{sym}}\left(u-\lambda v-w_{0}\right)\right|^{2} & \leq C_{H}(\Omega)\left(\int_{\Omega}\left|\nabla^{\mathrm{a}}(u-\lambda v)\right|^{2}+\int_{\Omega}|\nabla \cdot(u-\lambda v)|^{2}\right) \\
& \leq N C_{H}(\Omega) \bar{K}(\Omega)^{-1}\left\|\nabla^{\mathrm{sym}} u\right\|_{L^{2}(\Omega)}^{2} .
\end{aligned}
$$

Without loss of generality, we may assume $w_{0}=0$ : if this is not the case, replace $v$ by $v+w_{0} / \lambda$, which is still an element of $V_{\Sigma}$. So we know that there exists $v \in V_{\Sigma}$ such that

$$
\int_{\Omega}\left|\nabla^{\mathrm{sym}}(u-\lambda v)\right|^{2} \leq N C_{H}(\Omega) \bar{K}(\Omega)^{-1}\left\|\nabla^{\mathrm{sym}} u\right\|_{L^{2}(\Omega)}^{2} .
$$

Then

$$
\lambda^{2} \int_{\Omega}\left|\nabla^{\mathrm{sym}} v\right|^{2} \leq 2\left[N C_{H}(\Omega) \bar{K}(\Omega)^{-1}+1\right]\left\|\nabla^{\mathrm{sym}} u\right\|_{L^{2}(\Omega)}^{2} .
$$

Recalling definition (11), we conclude that

$$
|\Omega| \lambda^{2} \leq\left[N C_{H}(\Omega) \bar{K}(\Omega)^{-1}+1\right] G(\Omega)^{-1}\left\|\nabla^{\mathrm{sym}} u\right\|_{L^{2}(\Omega)}^{2} .
$$

This combined with (18) concludes the proof.

\section{Estimates of Grad's Number}

We now proceed to give some estimates from below for $G(\Omega)$ under the assumption that $\Omega$ is not axisymmetric. First of all, we give a precise definition, accompanied by a simple lemma, whose proof is omitted.

Definition - Lemma 1. Let $\Omega$ be a smooth bounded open subset of $\mathbb{R}^{N}, N \geq 2$. By abuse of language, we say that $\Omega$ is axisymmetric if and only if there exists a nontrivial rigid motion $R$ which is tangent to $\partial \Omega$; or equivalently, which satisfies

$$
\forall t \in \mathbb{R}, \quad \mathrm{e}^{t R} \Omega=\Omega
$$

or, equivalently, which satisfies

$$
\exists t_{0}>0 ; \forall t \in\left[0, t_{0}\right], \quad \mathrm{e}^{t R} \Omega=\Omega .
$$

Here $\mathrm{e}^{t R}$ is the isometry defined via

$$
\frac{\mathrm{d}}{\mathrm{d} t} \mathrm{e}^{t R}(x)=R \mathrm{e}^{t R}(x),
$$

and we use the shorthand $\mathrm{e}^{t R} x=\mathrm{e}^{t R}(x)$.

Next, let us recall some useful concepts from the theory of mass transportation, or Monge-Kantorovich minimization problems. Whenever $\mu$ is a probability measure on $\mathbb{R}^{N}$ and $T: \mathbb{R}^{N} \rightarrow \mathbb{R}^{N}$ is a measurable map, one defines the image measure $T \# \mu$ of $\mu$ by $T$ via the identity

$$
T \# \mu[A]=\mu\left[T^{-1}(A)\right] .
$$


Whenever $\mu$ and $\nu$ are two probability measures on $\mathbb{R}^{N}$, and $p \geq 1$ is given, one can define the Wasserstein distance of order $p$ between $\mu$ and $\nu$ by the formula

$$
W_{p}(\mu, \nu)=\inf _{\pi \in \Pi(\mu, \nu)}\left(\int_{\mathbb{R}^{N} \times \mathbb{R}^{N}}|x-y|^{p} \mathrm{~d} \pi(x, y)\right)^{1 / p},
$$

where $\Pi(\mu, \nu)$ stands for the set of all probability measures on $\mathbb{R}^{N} \times \mathbb{R}^{N}$ with marginals $\mu$ and $\nu$. In other words, $\pi$ belongs to $\Pi(\mu, \nu)$ if and only if for all bounded continuous functions $\varphi, \psi$ on $\mathbb{R}^{N}$,

$$
\int_{\mathbb{R}^{N} \times \mathbb{R}^{N}}[\varphi(x)+\psi(y)] \mathrm{d} \pi(x, y)=\int_{\mathbb{R}^{N}} \varphi \mathrm{d} \mu+\int_{\mathbb{R}^{N}} \psi \mathrm{d} \nu .
$$

From definition (21) one easily checks the convexity of $W_{p}^{p}$ with respect to $\mu$ and $\nu$. An important thing to know is that when $\mu$ and $\nu$ are absolutely continuous with respect to Lebesgue measure, then we have the equivalent definition

$$
W_{p}(\mu, \nu)=\inf _{T \# \mu=\nu}\left(\int_{\mathbb{R}^{N}}|x-T(x)|^{p} \mathrm{~d} \mu(x)\right)^{1 / p},
$$

where the infimum is taken over all maps $T: \mathbb{R}^{N} \rightarrow \mathbb{R}^{N}$ such that the image measure of $\mu$ by $T$ coincides with $\nu$. This and much more background on Wasserstein distances can be found in [17] for instance.

In the sequel, we shall use Wasserstein distances with particular probability measures, which will be of the form

$$
\mathcal{L}_{\Omega}=\frac{1_{\Omega}}{|\Omega|} \mathcal{L}
$$

where $\mathcal{L}$ stands for the Lebesgue measure on $\mathbb{R}^{N}$.

We can now state our main estimates. We shall use the standard notation

$$
\operatorname{dist}(x, A)=\inf _{y \in A}|x-y| \text {. }
$$

Proposition 5. Let $\Omega$ be a smooth bounded open subset of $\mathbb{R}^{N}$. Then, $G(\Omega)>0$ if and only if $\Omega$ is not axisymmetric. Moreover, for any $T>0$ one has the estimates

$$
G(\Omega) \geq \frac{1}{2|\Omega| P(\Omega)} \frac{\mathrm{e}^{-2 T}}{T^{3}} \inf _{R \in \mathcal{R}_{1}} \int_{0}^{T} W_{2}\left(\mathcal{L}_{\Omega}, \mathcal{L}_{\mathrm{e}^{t R} \Omega}\right)^{2} \mathrm{~d} t
$$

and

$$
G(\Omega) \geq \frac{1}{2|\Omega| P(\Omega)} \frac{\mathrm{e}^{-2 T}}{T^{3}} \inf _{R \in \mathcal{R}_{1}} \int_{0}^{T} \int_{\Omega} \operatorname{dist}\left(\mathrm{e}^{t R} x, \Omega\right)^{2} \mathrm{~d} x \mathrm{~d} t
$$

where $\mathcal{R}_{1}$ is the set of all rigid motions on $\mathbb{R}^{N}$ of the form $R(x)=\Sigma x+b$, with $|\Sigma|=1$, and $P(\Omega)$ is the Poincaré-Wirtinger constant, defined as the smallest admissible constant in the functional inequality

$$
\|f-\langle f\rangle\|_{L^{2}(\Omega)}^{2} \leq P(\Omega)\|\nabla f\|_{L^{2}(\Omega)}^{2} .
$$

Moreover, when $N=2$ or $N=3$, a simplified lower bound can be given as follows. Define the center of mass $g$ of $\Omega$ by

$$
g=\frac{1}{|\Omega|} \int_{\Omega} x \mathrm{~d} x
$$


Case $N=2$ : Define $\Omega_{\theta}$ as the image of $\Omega$ by the rotation of angle $\theta$ around $g$, and construct $\Omega^{\mathrm{sym}}$ by symmetrizing $\Omega$ around $g$ :

$$
\Omega^{\mathrm{sym}}=\bigcup_{0 \leq \theta \leq 2 \pi} \Omega_{\theta}
$$

Further define the probability measure $\mathcal{L}_{\Omega}^{\text {sym }}$ by symmetrization of $\mathcal{L}_{\Omega}$,

$$
\mathcal{L}_{\Omega}^{\mathrm{sym}}=\frac{1}{2 \pi} \int_{0}^{2 \pi} \mathcal{L}_{\Omega_{\theta}} \mathrm{d} \theta .
$$

Then there exists a numeric, explicit constant $K$ such that

$$
\begin{aligned}
G(\Omega) & \geq \frac{K}{|\Omega| P(\Omega)} \frac{1}{2 \pi} \int_{0}^{2 \pi} W_{2}\left(\mathcal{L}_{\Omega}, \mathcal{L}_{\Omega_{\theta}}\right)^{2} \mathrm{~d} \theta \\
& \geq \frac{K}{|\Omega| P(\Omega)} W_{2}\left(\mathcal{L}_{\Omega}, \mathcal{L}_{\Omega}^{\mathrm{sym}}\right)^{2} \\
& \geq \frac{K}{|\Omega| P(\Omega)} \int_{\Omega^{\mathrm{sym}} \backslash \Omega} \operatorname{dist}(y, \Omega)^{2} \mathrm{~d} \mathcal{L}_{\Omega}^{\mathrm{sym}}(y) .
\end{aligned}
$$

Case $N=3$ : For any $\sigma \in S^{2}$ define $\Delta_{\sigma}$ as the line going through $g$ and directed by $\sigma$. Then define $\Omega_{\theta}^{\sigma}$ as the image of $\Omega$ by the rotation of angle $\theta$ around the axis $\Delta_{\sigma}$, and define $\Omega^{\mathrm{sym} ; \sigma}, \mathcal{L}_{\Omega}^{\mathrm{sym} ; \sigma}$ by symmetrization of $\Omega$ and $\mathcal{L}_{\Omega}$ respectively:

$$
\Omega^{\mathrm{sym} ; \sigma}=\bigcup_{0 \leq \theta \leq 2 \pi} \Omega_{\theta}^{\sigma}, \quad \mathcal{L}_{\Omega}^{\mathrm{sym} ; \sigma}=\frac{1}{2 \pi} \int_{0}^{2 \pi} \mathcal{L}_{\Omega_{\theta}^{\sigma}} \mathrm{d} \theta .
$$

Then there exists a numeric, explicit constant $K$ such that

$$
\begin{aligned}
G(\Omega) & \geq \frac{K}{|\Omega| P(\Omega)} \inf _{\sigma \in S^{2}} \frac{1}{2 \pi} \int_{0}^{2 \pi} W_{2}\left(\mathcal{L}_{\Omega}, \mathcal{L}_{\Omega_{\theta}^{\sigma}}\right)^{2} \mathrm{~d} \theta \\
& \geq \frac{K}{|\Omega| P(\Omega)} \inf _{\sigma \in S^{2}} W_{2}\left(\mathcal{L}_{\Omega}, \mathcal{L}_{\Omega}^{\mathrm{sym} ; \sigma}\right)^{2} \\
& \geq \frac{K}{|\Omega| P(\Omega)} \inf _{\sigma \in S^{2}} \int_{\Omega^{\mathrm{sym} ; \sigma} \backslash \Omega} \operatorname{dist}(y, \Omega)^{2} \mathrm{~d} \mathcal{L}_{\Omega}^{\mathrm{sym} ; \sigma}(y) .
\end{aligned}
$$

\section{Remarks.}

1. Note that $\mathcal{L}_{\Omega}^{\mathrm{sym}} \neq \mathcal{L}_{\Omega^{\mathrm{sym}}}$ !!

2. Of course, in dimension $2, \Omega$ is axisymmetric if and only if $\Omega=\Omega^{\text {sym }}$, which is equivalent to $\mathcal{L}_{\Omega}=\mathcal{L}_{\Omega}^{\mathrm{sym}}$. Similarly, in dimension $3, \Omega$ is axisymmetric if and only if there exists $\sigma \in S^{2}$ such that $\Omega=\Omega^{\text {sym; } \sigma}$, which is equivalent to $\mathcal{L}_{\Omega}=\mathcal{L}_{\Omega}^{\text {sym; } \sigma}$. The bounds (28) and (31) are of course extremely simple, but sometimes the bounds (27) and (30) are much more precise. We shall discuss this at the end of the section.

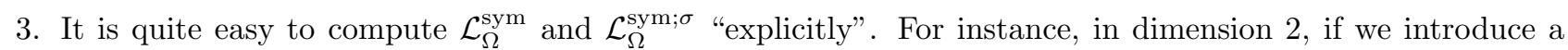
system of polar coordinates $(r, \theta)$ with center $g$, then the density of $\mathcal{L}_{\Omega}^{\text {sym }}$ at a point $\left(r_{0}, \theta_{0}\right)$ is given by

$$
\frac{1}{2 \pi}\left|\left\{\theta \in[0,2 \pi] ;\left(r_{0}, \theta\right) \in \Omega\right\}\right| .
$$


A similar expression can be derived in dimension 3 if one introduces a system of cylindrical coordinates with vertical direction $\sigma$.

Proof of Proposition 5. It is immediate to show that $G(\Omega)=0$ if $\Omega$ is axisymmetric. Conversely, let us show that if $\Omega$ is not axisymmetric, then $G(\Omega)>0$. Assume by contradiction that $G(\Omega)=0$, so there exists a sequence $\Sigma_{n} \in U A_{N}, v_{n} \in V_{\Sigma_{n}}$ such that $\left\|\nabla^{\mathrm{sym}} v_{n}\right\|_{L^{2}} \longrightarrow 0$ as $n \rightarrow \infty$. Then $\left\|\nabla v_{n}\right\|_{L^{2}}$ is bounded, since $\left\|\nabla^{\mathrm{a}} v_{n}\right\|$ is also bounded. By Poincaré-Wirtinger's inequality $(25)$ the sequence $\left(v_{n}-\left\langle v_{n}\right\rangle\right)$ is bounded in $H^{1}\left(\Omega ; \mathbb{R}^{N}\right)$. Up to extraction of a subsequence, we may assume that it converges towards some $v$, weakly in $H^{1}\left(\Omega ; \mathbb{R}^{N}\right)$. Since $U A_{N}$ is compact, we may also assume that $\Sigma_{n}$ converges towards some $\Sigma \in U A_{N}$ as $n \rightarrow \infty$. Then it is easily checked that $v \in V_{\Sigma}$ and $\nabla^{\operatorname{sym}} v=0$, so in fact $\nabla v=\Sigma$ and $v$ is a rigid motion. By Lemma 1, $\Omega$ is axisymmetric.

Next, we turn to estimates (23) and (24). Let $\Sigma \in U A_{N}$, and let $v \in V_{\Sigma}$. Define the rigid motion $R$ by

$$
R(x)=\Sigma x+b,
$$

where $b \in \mathbb{R}^{N}$ will be chosen later on. Introduce the exponential maps, solutions of

$$
\left\{\begin{array}{l}
\frac{\mathrm{d}}{\mathrm{d} t} \mathrm{e}^{t v}(x)=v\left(\mathrm{e}^{t v}(x)\right), \\
\frac{\mathrm{d}}{\mathrm{d} t} \mathrm{e}^{t R}(x)=R\left(\mathrm{e}^{t R}(x)\right) .
\end{array}\right.
$$

Then,

$$
\begin{aligned}
\frac{\mathrm{d}}{\mathrm{d} t}\left|\mathrm{e}^{t v}(x)-\mathrm{e}^{t R}(x)\right| & \leq\left|v\left(\mathrm{e}^{t v}(x)\right)-R\left(\mathrm{e}^{t R}(x)\right)\right| \\
& \leq\left|v\left(\mathrm{e}^{t v}(x)\right)-R\left(\mathrm{e}^{t v}(x)\right)\right|+\left|R\left(\mathrm{e}^{t v}(x)\right)-R\left(\mathrm{e}^{t R}(x)\right)\right| .
\end{aligned}
$$

Since the Lipschitz norm of $R$ is $|\Sigma|=1$, the last term is bounded by $\left|\mathrm{e}^{t v}(x)-\mathrm{e}^{t R}(x)\right|$, and by Gronwall's lemma

$$
\left|\mathrm{e}^{t v}(x)-\mathrm{e}^{t R}(x)\right| \leq \mathrm{e}^{t} \int_{0}^{t}\left|v\left(\mathrm{e}^{s v}(x)\right)-R\left(\mathrm{e}^{s v}(x)\right)\right| \mathrm{d} s .
$$

Then, a crude estimate yields

$$
\frac{1}{T} \int_{0}^{T}\left|\mathrm{e}^{t v}(x)-\mathrm{e}^{t R}(x)\right|^{2} \mathrm{~d} t \leq T \mathrm{e}^{2 T} \int_{0}^{T}\left|v\left(\mathrm{e}^{s v}(x)\right)-R\left(\mathrm{e}^{s v}(x)\right)\right|^{2} \mathrm{~d} s .
$$

Integrating over $\Omega$, we find

$$
\frac{1}{T} \int_{0}^{T} \int_{\Omega}\left|\mathrm{e}^{t v}(x)-\mathrm{e}^{t R}(x)\right|^{2} \mathrm{~d} x \mathrm{~d} t \leq T \mathrm{e}^{2 T} \int_{0}^{T} \int_{\Omega}\left|v\left(\mathrm{e}^{t v}(x)\right)-R\left(\mathrm{e}^{t v}(x)\right)\right|^{2} \mathrm{~d} x \mathrm{~d} t .
$$

Next, since $v$ is divergence-free, we know that the image measure of the Lebesgue measure on $\Omega$ by the map $\mathrm{e}^{t v}$ is just the Lebesgue measure. So the right-hand side of (33) is in fact

$$
T \mathrm{e}^{2 T} \int_{0}^{T} \int_{\Omega}|v(x)-R(x)|^{2} \mathrm{~d} x \mathrm{~d} t=T^{2} \mathrm{e}^{2 T} \int_{\Omega}|v(x)-R(x)|^{2} \mathrm{~d} x .
$$

Now we choose $b$ in such a way that $\langle v-R\rangle=0$. Combining (33) with Poincaré's inequality (25) we obtain

$$
\frac{1}{T} \int_{0}^{T} \int_{\Omega}\left|\mathrm{e}^{t v}(x)-\mathrm{e}^{t R}(x)\right|^{2} \mathrm{~d} x \mathrm{~d} t \leq P(\Omega) T^{2} \mathrm{e}^{2 T} \int_{\Omega}|\nabla v(x)-\Sigma|^{2} \mathrm{~d} x .
$$


But $\nabla^{\mathrm{a}} v=\Sigma$ ! So this inequality can be rewritten as

$$
\frac{1}{T} \int_{0}^{T} \int_{\Omega}\left|\mathrm{e}^{t v}(x)-\mathrm{e}^{t R}(x)\right|^{2} \mathrm{~d} x \mathrm{~d} t \leq P(\Omega) T^{2} \mathrm{e}^{2 T} \int_{\Omega}\left|\nabla^{\mathrm{sym}} v\right|^{2} \mathrm{~d} x .
$$

Now, since $v$ is tangent to the boundary of $\Omega$, it follows that for all $x \in \Omega$, one has $\mathrm{e}^{t v}(x) \in \Omega$. Thus the left-hand side of (34) is bounded below by

$$
\frac{1}{T} \int_{0}^{T} \int_{\Omega} \operatorname{dist}\left(\mathrm{e}^{t R}(x), \Omega\right)^{2} \mathrm{~d} x \mathrm{~d} t
$$

which proves (24).

To prove (23), start again from (34), and use the fact that $\mathrm{e}^{t v}$ is a measure-preserving diffeomorphism of $\Omega$ (with inverse $\mathrm{e}^{-t v}$ ) to get

$$
\int_{\Omega}\left|\mathrm{e}^{t v}(x)-\mathrm{e}^{t R}(x)\right|^{2} \mathrm{~d} x=\int_{\Omega}\left|x-\mathrm{e}^{t R} \circ \mathrm{e}^{-t v}(x)\right|^{2} \mathrm{~d} x
$$

next note that $\left(\mathrm{e}^{-t R} \circ \mathrm{e}^{-t v}\right) \# \mathcal{L}_{\Omega}=\mathrm{e}^{-t R} \# \mathcal{L}_{\Omega}=\mathcal{L}_{\mathrm{e}^{-t R} \Omega}$, because $\mathrm{e}^{-t R}$ preserves Lebesgue measure on $\mathbb{R}^{N}$. Apply definition (22) to conclude.

We now proceed to establish the simplified expressions when $N=2$ or 3 . We shall only treat the case $N=2$ since the case $N=3$ is exactly similar. Without loss of generality, we assume $g=0$. Let $R$ be a rigid motion of $\mathbb{R}^{2}$ of the form $R(x)=\Sigma x+b$, with $|\Sigma|=1$. Then $\mathrm{e}^{\sqrt{2} \theta R}$ is the rotation of angle $\theta$ around a certain point $x_{0}$. One can write

$$
\mathrm{e}^{\sqrt{2} \theta R} x=x_{0}+\rho_{\theta}\left(x-x_{0}\right)
$$

where $\rho_{\theta}$ stands for the rotation of angle $\theta$ around 0 . Note that $\mathrm{e}^{2 \sqrt{2} \pi R}$ is the identity. We shall show that for any $\theta \in[0,2 \pi]$,

$$
\int_{\Omega}\left|\mathrm{e}^{\sqrt{2} \theta v}(x)-\mathrm{e}^{\sqrt{2} \theta R}(x)\right|^{2} \mathrm{~d} x \geq \int_{\Omega}\left|\mathrm{e}^{\sqrt{2} \theta v}(x)-\rho_{\theta}(x)\right|^{2} \mathrm{~d} x ;
$$

in other words, the left-hand side of (35) can only become smaller if we impose $R g=0$. This will prove that we only need to consider the symmetrization around $g$.

To prove (35) we write, using the notation $I$ for the identity,

$$
\begin{gathered}
\int_{\Omega}\left|\mathrm{e}^{\sqrt{2} \theta v}(x)-\mathrm{e}^{\sqrt{2} \theta R}(x)\right|^{2} \mathrm{~d} x=\int_{\Omega}\left|\mathrm{e}^{\sqrt{2} \theta v}(x)-x_{0}-\rho_{\theta}\left(x-x_{0}\right)\right|^{2} \mathrm{~d} x \\
=\int_{\Omega}\left|\mathrm{e}^{\sqrt{2} \theta v}(x)-\rho_{\theta}(x)\right|^{2} \mathrm{~d} x+|\Omega|\left|\left(I-\rho_{\theta}\right) x_{0}\right|^{2}-2\left\langle\int_{\Omega}\left[\mathrm{e}^{\sqrt{2} \theta v}(x)-\rho_{\theta}(x)\right] \mathrm{d} x,\left(I-\rho_{\theta}\right) x_{0}\right\rangle .
\end{gathered}
$$

Then we notice that, since $\mathrm{e}^{\sqrt{2} \theta v}$ is a measure-preserving map from $\Omega$ into itself,

$$
\int_{\Omega} \mathrm{e}^{\sqrt{2} \theta v}(x) \mathrm{d} x=\int_{\Omega} x \mathrm{~d} x=0,
$$

while

$$
\int_{\Omega} \rho_{\theta}(x) \mathrm{d} x=\rho_{\theta}\left(\int_{\Omega} x \mathrm{~d} x\right)=\rho_{\theta}(0)=0 .
$$


Thus

$$
\int_{\Omega}\left|\mathrm{e}^{\sqrt{2} \theta v}(x)-\mathrm{e}^{\sqrt{2} \theta R}(x)\right|^{2} \mathrm{~d} x=\int_{\Omega}\left|\mathrm{e}^{\sqrt{2} \theta v}(x)-\rho_{\theta}(x)\right|^{2} \mathrm{~d} x+|\Omega|\left|\left(I-\rho_{\theta}\right) x_{0}\right|^{2},
$$

which proves (35).

Remark. A reader familiar with mass transportation may have recognized the elementary argument used to prove that the Monge-Kantorovich transportation problem with exponent 2 commutes with translations.

From (35) and (23) we deduce (26). Then, (27) follows by convexity of $W_{2}^{2}$. Next, by symmetry of the Wasserstein distances,

$$
W_{2}\left(\mathcal{L}_{\Omega}, \mathcal{L}_{\Omega}^{\text {sym }}\right)^{2}=\inf _{T \# \mathcal{L}_{\Omega}^{\text {sym }}=\mathcal{L}_{\Omega}} \int_{\Omega^{\text {sym }}}|x-T(x)|^{2} \mathrm{~d} \mathcal{L}_{\Omega}^{\text {sym }}(x) .
$$

Of course, if $T \# \mathcal{L}_{\Omega}^{\text {sym }}=\mathcal{L}_{\Omega}$, then necessarily $T\left(\Omega^{\text {sym }}\right) \subset \Omega$, so that $|x-T(x)|$ in the integrand is greater than $\operatorname{dist}(x, \Omega)$. This proves $(28)$.

We conclude this section with some simple remarks about practical computations. As we said before, formulas (28) and (31) are very convenient and can easily be computed numerically. On the other hand, if $\Omega$ is very close to be axisymmetric, these lower bounds may become much smaller than $G(\Omega)$. Consider for instance the situation where $\Omega$ is a very slightly elongated ellipse in the plane, something like

$$
E=\left\{\left(x_{1}, x_{2}\right) \in \mathbb{R}^{2} ; x_{1}^{2}+\frac{x_{2}^{2}}{1+\varepsilon} \leq 1\right\}, \quad \Omega=\frac{E}{\sqrt{|E|}}
$$

(here we have normalized the volume of $\Omega$ to unity) for small $\varepsilon$. Then the symmetrized Lebesgue measure of $\Omega$ takes value 1 within a disc centered at 0 , with radius approximately 1 , and then decreases to 0 on a thin shell of thickness $O(\varepsilon)$. One can then show that

$$
\int_{\Omega^{\text {sym }}} \operatorname{dist}(x, \Omega)^{2} \mathrm{~d} \mathcal{L}_{\Omega}^{\text {sym }}(x)=O\left(\varepsilon^{3}\right) .
$$

On the other hand, from elementary mass transportation theory,

$$
W_{2}\left(\mathcal{L}_{\Omega}, \mathcal{L}_{\Omega}^{\mathrm{sym}}\right)^{2} \text { is at least of the order of } \varepsilon^{2} .
$$

A way to arrive at (36) is to apply the inequality

$$
W_{2}\left(\mathcal{L}_{\Omega}, \mathcal{L}_{\Omega}^{\text {sym }}\right)^{2} \geq W_{1}\left(\mathcal{L}_{\Omega}, \mathcal{L}_{\Omega}^{\text {sym }}\right)^{2},
$$

and then to use the identity

$$
W_{1}(\mu, \nu)=W_{1}\left([\mu-\nu]_{+},[\mu-\nu]_{-}\right) .
$$

The idea behind (37) is that when the cost function is a distance, then all the mass which can stay in place in the transportation process (the shared mass between $\mu$ and $\nu$ ) can be required to do so, and this does not affect the value of the optimal cost. Note that in the right-hand side of (37), we have extended the definition of $W_{1}$ to arbitrary nonnegative measures with a common mass, not necessarily normalized to 1 . Then it is easy to convince oneself that transporting $\left[\mathcal{L}_{\Omega}-\mathcal{L}_{\Omega}^{\text {sym }}\right]_{+}$onto $\left[\mathcal{L}_{\Omega}-\mathcal{L}_{\Omega}^{\text {sym }}\right]_{-}$with cost $c(x, y)=|x-y|$ requires at least a cost of order $\varepsilon$, because at least a mass of order $\varepsilon$ has to be moved on a distance of order 1 . 


\section{Some Remarks about the AXisymmetric CASE}

What becomes of Theorem 1 when $\Omega$ is axisymmetric? The question is of interest for our problem of relaxation to thermodynamical equilibrium, since it is natural to ask what happens if the gas is enclosed in a cylinder. Let us concentrate on the case $N=3$. First, one should make the distinction between a cylinder with only one axis of symmetry, and a spherically symmetric domain. Recall that if a domain $\Omega \subset \mathbb{R}^{3}$ admits two nonparallel axes of symmetry, then it is spherically symmetric around some point.

If $\Omega$ has spherical symmetry, then we should just be content with inequality (1). The case of a cylinder with a unique axis of symmetry, is a little bit more involved. Without loss of generality, assume that the axis of symmetry of $\Omega$ passes through $g=0$ and is directed by $\omega \in S^{2}$. Introduce the orthogonal decomposition

$$
\langle\nabla \wedge u\rangle=\lambda \sigma+\mu \omega, \quad \sigma \perp \omega .
$$

Introduce a rigid rotation $R$ around $\omega$, of the form $R(x)=\mu \omega \wedge x$; then

$$
\langle\nabla \wedge(u-R)\rangle=\lambda \sigma, \quad \nabla^{\mathrm{sym}}(u-R)=\nabla^{\mathrm{sym}} u .
$$

Since $R$ is tangent to the boundary of $\Omega$, one can repeat the proof of Theorem 3 and find

$$
\left\|\nabla^{\mathrm{sym}} u\right\|_{L^{2}(\Omega)}^{2} \geq K(\Omega) \inf _{R \in \mathcal{R}_{\omega}(\Omega)}\|\nabla(u-R)\|_{L^{2}(\Omega)}^{2},
$$

where $\mathcal{R}_{\omega}(\Omega)$ stands for the set of all rotations with axis $\omega$. Moreover, $R$ is optimal in the right-hand side of (38) if and only if $\nabla \wedge R$ is the average of the orthogonal projection of $\nabla \wedge u$ onto $\omega$, and the constant $K(\Omega)$ is proportional to

$$
G_{\omega}(\Omega)=\inf _{\sigma \in S^{2} ; \sigma \cdot \omega=0} \inf _{v \in V_{\sigma}}\left\|\nabla^{\mathrm{sym}} u\right\|_{L^{2}(\Omega)}^{2}
$$

To summarize the situation in dimension 3: if $\Omega$ is a ball, then inequality (1) only shows that $\nabla^{\mathrm{sym}} u$ controls the departure of $u$ from being a rigid motion; while if $\Omega$ is a cylinder with only axis $\omega$, then $\nabla^{\text {sym }} u$ controls the departure of $u$ from being a rigid motion with axis $\omega$. This is perfectly consistent with the context of trend to equilibrium for the Boltzmann equation, because the last indeterminacy about $u$ will be compensated for by 3 additional conservation laws (angular momentum) in the case of a ball, and by one additional conservation law ( $\omega$-component of the angular momentum) in the case of a cylinder with only axis $\omega$.

\section{APPENDIX}

Here we reproduce the elegant proof, communicated to us by Druet, of estimate (10) for a convex domain with $C_{H}=1$. It is based on the elementary identity

$$
\left|\nabla^{\mathrm{sym}} u\right|^{2}-\left|\nabla^{\mathrm{a}} u\right|^{2}=(\nabla \cdot u)^{2}+\nabla \cdot[(u \cdot \nabla) u-u(\nabla \cdot u)]
$$

with the usual notation

$$
u \cdot \nabla=\sum_{i=1}^{N} u_{i} \frac{\partial}{\partial x_{i}}
$$

In fact, identity (39) is well-known in the theory of the Korn inequality because it provides an elementary proof of (3) when $\Gamma=\partial \Omega$ : indeed, when $u=0$ on $\partial \Omega$, it implies, by divergence theorem,

$$
\int_{\Omega}\left|\nabla^{\mathrm{a}} u\right|^{2}=\int_{\Omega}\left|\nabla^{\mathrm{sym}} u\right|^{2}-\int_{\Omega}(\nabla \cdot u)^{2} \leq \int_{\Omega}\left|\nabla^{\mathrm{sym}} u\right|^{2} .
$$


Let us now turn to (10). The problem is somehow opposite since we have to control the symmetric part instead of the antisymmetric! Let $u$ be an arbitrary vector field $u \in H^{1}\left(\Omega ; \mathbb{R}^{N}\right)$, tangent to the boundary. Again, identity (39) and use of the divergence theorem imply (remember that $u \cdot n=0$ on the boundary)

$$
\begin{aligned}
\int_{\Omega}\left|\nabla^{\mathrm{sym}} u\right|^{2} & =\int_{\Omega}\left|\nabla^{\mathrm{a}} u\right|^{2}+\int_{\Omega}(\nabla \cdot u)^{2}+\int_{\partial \Omega}[(u \cdot \nabla) u-u(\nabla \cdot u)] \cdot n \\
& =\int_{\Omega}\left|\nabla^{\mathrm{a}} u\right|^{2}+\int_{\Omega}(\nabla \cdot u)^{2}+\int_{\partial \Omega}[(u \cdot \nabla) u] \cdot n .
\end{aligned}
$$

But, since $u$ is tangent to the boundary, $u \cdot \nabla$ is just the covariant derivative along $u$, so

$$
[(u \cdot \nabla) u] \cdot n=-(u \cdot \nabla n) \cdot u=-I_{\Omega}(u, u)
$$

where $I I_{\Omega}$ stands for the real-valued second fundamental form of $\Omega$ (see for instance [7], p. 217). A well-known property of the second fundamental form is that it is nonnegative as soon as $\Omega$ is convex. Thus in the end

$$
\int_{\Omega}\left|\nabla^{\mathrm{sym}} u\right|^{2}=\int_{\Omega}\left|\nabla^{\mathrm{a}} u\right|^{2}+\int_{\Omega}(\nabla \cdot u)^{2}-\int_{\partial \Omega} I I_{\Omega}(u, u) \leq \int_{\Omega}\left|\nabla^{\mathrm{a}} u\right|^{2}+\int_{\Omega}(\nabla \cdot u)^{2}
$$

which immediately implies (10) with $C_{H}=1$.

Serre has a slightly different argument (not more complicated), also based on (42), leading to the same result. We further note that the use of a trace theorem, combined with a Poincaré-like inequality, implies

$$
\int_{\partial \Omega}|u|^{2} \leq C \int_{\Omega}|\nabla u|^{2}
$$

and this together with (43) enables one to get estimates of $C_{H}$ when $\Omega$ is a $C^{2}$ perturbation of a convex set. The general case in which $\Omega$ is not close from a convex set looks much more difficult.

Even if the present paper is rather short, it benefited a lot from the kind advice of many colleagues who helped us make our ideas clear about the inequalities which we discussed above: in particular Guy Bouchitté, Yann Brenier, Philippe Ciarlet, Olivier Druet, Craig Evans, Giuseppe Geymonat, Étienne Ghys, Yves Meyer, Stefan Müller, Denis Serre, Bruno Sévennec, Jean-Claude Sikorav. It is a pleasure to warmly thank them all. The support of the European TMR contract "Asymptotic Methods in Kinetic Theory", ERB FMBX CT97 0157 is also acknowledged.

Dedication. This work is dedicated to the memory of Jacques-Louis Lions, whose contribution to the theory of Korn's inequality was both crucial and beautifully simple. It is also a tribute to the brilliant intuitions of Harold Grad in the theory of the Boltzmann equation. We are particularly glad to note that our arguments rest not only on ideas arising from elasticity theory and the kinetic theory of gases, but also from the field of mass transportation, which was once developed by Kantorovich for its links with economics, and later impulsed by Brenier for its connections with hydrodynamics. Here Korn's inequality appears as a beautiful link between all of these fields.

\section{REFERENCES}

[1] P.G. Ciarlet, Mathematical elasticity. Vol. I. Three-dimensional elasticity. Vol. II: Theory of plates. Vol. III: Theory of shells. North-Holland Publishing Co., Amsterdam (1988, 1997, 2000).

[2] D. Cioranescu, O.A. Oleinik and G. Tronel, On Korn's inequalities for frame type structures and junctions. C. R. Acad. Sci. Paris Sér. I Math. 309 (1989) 591-596.

[3] L. Desvillettes, Convergence to equilibrium in large time for Boltzmann and BGK equations. Arch. Rational Mech. Anal. 110 (1990) 73-91. 
[4] L. Desvillettes and C. Villani, On the trend to global equilibrium in spatially inhomogeneous entropy-dissipating systems: The Boltzmann equation. Work in progress.

[5] G. Duvaut and J.-L. Lions, Inequalities in mechanics and physics. Springer-Verlag, Berlin (1976). Translated from the French by C.W. John, Grundlehren der Mathematischen Wissenschaften, 219.

[6] K.O. Friedrichs, On the boundary-value problems of the theory of elasticity and Korn's inequality. Ann. Math. 48 (1947) 441-471.

[7] S. Gallot, D. Hulin and J. Lafontaine, Riemannian geometry, Second Edition. Springer-Verlag, Berlin (1990).

[8] J. Gobert, Une inégalité fondamentale de la théorie de l'élasticité. Bull. Soc. Roy. Sci. Liège 31 (1962) $182-191$.

[9] H. Grad, On Boltzmann's H-theorem. J. Soc. Indust. Appl. Math. 13 (1965) 259-277.

[10] C.O. Horgan, Korn's inequalities and their applications in continuum mechanics. SIAM Rev. 37 (1995) $491-511$.

[11] C.O. Horgan and L.E. Payne, On inequalities of Korn, Friedrichs and Babuska-Aziz. Arch. Rational Mech. Anal. 82 (1983) 165-179.

[12] R.V. Kohn, New integral estimates for deformations in terms of their nonlinear strains. Arch. Rational Mech. Anal. 78 (1982) $131-172$.

[13] A. Korn, Solution générale du problème d'équilibre dans la théorie de l'élasticité, dans le cas où les effets sont donnés à la surface. Ann. Fac. Sci. Univ. Toulouse 10 (1908) 165-269.

[14] J.A. Nitsche, On Korn's second inequality. RAIRO: Anal. Numér. 15 (1981) 237-248.

[15] V.A. Kondratiev and O.A. Oleinik, On Korn's inequalities. C. R. Acad. Sci. Paris Sér. I Math. 308 (1989) $483-487$.

[16] E.I. Ryzhak, Korn's constant for a parallelepiped with a free face or pair of faces. Math. Mech. Solids 4 (1999) 35-55.

[17] C. Villani, Topics in mass transportation. Preprint (2002).

[18] Y. Shizuta and K. Asano, Global solutions of the Boltzmann equation in a bounded convex domain. Proc. Japan Acad. Ser. A Math. Sci. 53 (1977) 3-5. 